Pacific Journal of Mathematics

THE COLLARS OF A RIEMANNIAN MANIFOLD AND STABLE 


\title{
THE COLLARS OF A RIEMANNIAN MANIFOLD AND STABLE ISOSYSTOLIC INEQUALITIES
}

\author{
JAMES J. HEBDA
}

\begin{abstract}
We define the $p$-dimensional collar $\operatorname{Col}_{p}(M, g)$ of a compact torsion-free Riemannian manifold $(M, g)$ to be the greatest lower bound of the masses of all the $p$-dimensional currents which represent non-trivial integral homology classes. When the cohomology ring of $M$ satisfies a certain non-degeneracy condition there is an inequality giving a lower bound on the volume of $(M, g)$ in terms of certain $p$-dimensional collars of $(M, g)$. This is a version of the stable isosystolic inequality using currents rather than singular homology.

In addition to deriving this version of the stable isosystolic inequality, we show for one class of manifolds that it is a sharp inequality.
\end{abstract}

TheOREM A. Let $(M, g)$ be a compact oriented n-dimensional Riemannian manifold with $H_{1}(M, Z) \cong Z$. Then

$$
\operatorname{Vol}(M, g) \geq \operatorname{Col}_{1}(M, g) \operatorname{Col}_{n-1}(M, g) \text {. }
$$

Furthermore, equality holds if and only if there is a Riemannian submersion of $(M, g)$ onto the circle of length $\operatorname{Col}_{1}(M, g)$ such that each level hypersurface (i.e. fiber) is a connected minimal submanifold of volume $\mathrm{Col}_{n-1}(M, g)$.

It is interesting to contrast Theorem A with Loewner's inequality [2], [8] which gives a lower bound on the area of a torus in terms of the length of the shortest non-contractible closed curve. In Loewner's theorem equality holds for a class of metrics which differ from one another by a constant multiple. Whereas in Theorem A equality can hold for many very different Riemannian metrics. As an example let $M=S^{1} \times S^{2}$. Certainly the equation $\operatorname{Vol}(M, g)=\operatorname{Col}_{1}(M, g) \operatorname{Col}_{2}(M, g)$ will hold for any of the various product metrics $g$. It also will hold for some non-product metrics. One of the latter can be constructed as follows. Let $S^{2}$ be given the canonical constant curvature metric, and let $f$ be a non-trivial orientation preserving isometry of $S^{2}$. Then the group of integers acts as a properly discontinuous group of isometries on the Riemannian product $R \times S^{2}$ by defining $n(t, x)=\left(t+n, f^{n}(x)\right)$ where $f^{n}$ is the $n$th iterate of $f$. The quotient space under this action is diffeomorphic to $M$. Hence the metric on $R \times S^{2}$ passes down to a non-product metric on $M$ for which (1) the 
projection on the first factor is a Riemannian submersion and (2) the level surfaces of the projection are connected minimal submanifolds. Thus by Theorem A the volume of this metric equals the product of the 1- and 2-dimensional collars.

It is easy to see that the cohomology rings of the complex and quaternionic projective spaces and of the product of spheres satisfy appropriate non-degeneracy conditions, and thus that the stable isosystolic inequalities hold for these spaces. (See Gromov [6], [7].) We show that the cohomology ring of $C P^{m} \times C P^{n}$ satisfies a strong non-degeneracy condition. Consequently the following theorem holds.

THEOREM B. Let $M=C P^{m} \times C P^{n}$. Given any positive integers $i_{1}, \ldots, i_{k}$ such that $i_{1}+\cdots+i_{k}=m+n$ there is a constant $C\left(i_{1}, \ldots, i_{k}\right)$ $>0$ such that

$$
\operatorname{Vol}(M, g) \geq C\left(i_{1}, \ldots, i_{k}\right) \operatorname{Col}_{2 i_{1}}(M, g) \cdots \operatorname{Col}_{2 i_{k}}(M, g)
$$

for each Riemannian metric $g$ on $M$.

There are analogous inequalities for the product of two quaternionic projective spaces and the product of two Cayley planes. This can be proved by modifications in the proof of Theorem B.

We conclude with an inequality bounding the first eigenvalue of the Laplacian of $(M, g)$ from above in terms of some of the collars of $(M, g)$.

Norms on forms and cohomology. Let $(M, g)$ be a connected compact oriented $n$-dimensional Riemannian manifold. The canonical volume form of $(M, g)$ will be denoted by $v_{g}$. Thus $\operatorname{Vol}(M, g)=\int_{M} v_{g}$ is the volume of $(M, g)$. The pointwise inner product of two $p$-forms $\omega$ and $\varphi$ on $M$ is the function $\langle\omega, \varphi\rangle$ defined by

$$
\omega \wedge * \varphi=\langle\omega, \varphi\rangle v_{g}
$$

where $*$ is the Hodge-star operator on forms. The corresponding pointwise norm of the $p$-form $\omega$ will be denoted by $|\omega|$. The following properties are well known $[5,10]$.

$$
\begin{array}{lll} 
& * 1=v_{g}, & \\
& * * \omega=(-1)^{(n-p) p} \omega & \text { for all } p \text {-forms } \omega, \\
& |* \omega|=|\omega| & \text { for all } p \text {-forms } \omega, \text { and, } \\
\text { (1) } & |\omega \wedge \varphi| \leq C_{1}(p, q ; n)|\omega||\varphi| & \text { for all } p \text {-forms } \omega
\end{array}
$$

and $q$-forms $\varphi$ where $C_{1}(p, q ; n)$ is a constant depending only on $p, q$, and $n$. 
Remark. Always

$$
C_{1}(p, q ; n) \leq\left(\begin{array}{c}
p+q \\
p
\end{array}\right)^{1 / 2}
$$

and if either $p$ or $q$ equals $0,1, n-1$, or $n$, then $C_{1}(p, q ; n)=1$ because in this case at least one of the two forms is simple. Clearly $C_{1}(p, q ; n)=$ $C_{1}(q, p ; n)$. See [5].

By integrating over $M$ one obtains the global inner product on forms:

$$
\langle\langle\omega, \varphi\rangle\rangle=\int_{M}\langle\omega, \varphi\rangle v_{g} \quad \text { for } p \text {-forms } \omega \text { and } \varphi \text {. }
$$

By Hodge Theory and the de Rham isomorphism, every real cohomology class $\alpha \in H^{p}(M, R)$ is uniquely represented by a harmonic $p$-form. We define the inner product of two cohomology classes $\alpha, \beta \in H^{p}(M, R)$ by the formula

$$
\langle\langle\alpha, \beta\rangle\rangle=\langle\langle\omega, \varphi\rangle\rangle
$$

where $\omega$ and $\varphi$ are the harmonic $p$-forms representing $\alpha$ and $\beta$ respectively. || || will denote the corresponding norms for forms and for cohomology classes.

The comass of a $p$-form $\omega$ at the point $x$ in $M$ is

$$
\left|\omega_{x}\right|_{0}=\sup \left\{\omega_{x}(X): X \in \wedge^{p} T_{x} M \text { is a simple } p \text {-vector of length } \leq 1\right\},
$$

and the comass of $\omega$ is

$$
\|\omega\|_{0}=\sup \left\{\left|\omega_{x}\right|_{0}: x \in M\right\}
$$

REMARK. One always has the following inequalities between the pointwise norm and the comass of $\omega$ which are valid at every point of $M$ :

$$
\begin{aligned}
& |\omega|_{0} \leq|\omega| \\
& |\omega| \leq C_{2}(p ; n)|\omega|_{0}
\end{aligned}
$$

where $C_{2}(p ; n) \leq\left(\begin{array}{c}n \\ p\end{array}\right)$ is a constant depending only on $n$ and $p$. Furthermore, $C_{2}(p ; n)=1$ if $p$ equals $0,1, n-1$, or $n$, and $C_{2}(p ; n)=$ $C_{2}(n-p ; n)$. See $[5,11]$.

Norms on homology. A $p$-dimensional current is a continuous linear functional on the vector space of all $p$-forms endowed with the $C^{\infty}$-topology. The mass $|T|$ of a $p$-dimensional current $T$ is defined by

$$
|T|=\sup \left\{T(\omega): \omega \text { is a } p \text {-form with }\|\omega\|_{0} \leq 1\right\} .
$$


Recall that if $T$ is integration over a submanifold of $M$, then $|T|$ is the volume of the submanifold, and that if $T$ is a singular chain, then $|T|$ is the mass of $T$ defined in Gromov [7]. The boundary of the $p$-dimensional current $T$ is the ( $p$-1)-dimensional current $\partial T$ satisfying $\partial T(\omega)=T(d \omega)$ for every $(p-1)$-form $\omega$. This turns the collection of all currents into a differential complex whose homology is canonically isomorphic to the real homology of $M$ [9]. We define the mass of a real homology class $a \in H_{p}(M, R)$ by

$$
\|a\|=\inf \{|T|: T \in a\} .
$$

This definition of mass compares with Gromov's $\mathrm{Vol}_{R}$ as follows. $\|a\| \leq \operatorname{Vol}_{R}(a)$ at least when $a$ is an integral homology class.

The norms defined in this and the preceding section all depend upon the Riemannian metric $g$.

Duality. $D(a) \in H^{n-p}(M, R)$ denotes the Poincaré dual class of $a \in H_{p}(M, R)$. Likewise, $D(\alpha) \in H_{n-p}(M, R)$ denotes the Poincaré dual of $\alpha \in H^{p}(M, R)$. Thus, if $a \cdot b \in H_{p+q-n}(M, R)$ is the intersection class correspondinig to $a \in H_{p}(M, R)$ and $b \in H_{q}(M, R)$, then $D(a \cdot b)=$ $D(a) \cup D(b)$ where $\cup$ is the cup product.

LeMMA 1. For every $a \in H_{p}(M, R)$ and every $(n-p)$-form $\omega$ representing $D(a)$,

$$
\|a\| \leq C_{2}(p ; n) \int_{M}|\omega| v_{g}
$$

Proof. Define a $p$-dimensional current $T$ by the formula

$$
T(\varphi)=\int_{M} \omega \wedge \varphi
$$

for every $p$-form $\varphi$. Then $T \in a$. It follows from (2) that $|\varphi| \leq C_{2}(p ; n)$ for every $p$-form $\varphi$ satisfying $\|\varphi\|_{0} \leq 1$. Hence, if $\|\varphi\|_{0} \leq 1$,

$$
\begin{aligned}
T(\varphi) & =\int_{M} \omega \wedge \varphi=\int_{M}\langle * \omega, \varphi\rangle v_{g} \\
& \leq \int_{M}|* \omega||\varphi| v_{g} \leq C_{2}(p ; n) \int_{M}|\omega| v_{g}
\end{aligned}
$$

where we have integrated Schwarz's inequality for the pointwise inner product on forms and used $|* \omega|=|\omega|$. Thus

$$
\|a\| \leq|T| \leq C_{2}(p ; n) \int_{M}|\omega| v_{g} .
$$


LEMMA 2. If $a \in H_{n-p}(M, R)$ and $b \in H_{n-q}(M, R)$, then

$$
\|D(a)\|\|D(b)\| \geq C_{3}(p, q ; n)\|a \cdot b\|
$$

where $C_{3}(p, q ; n)=\left(C_{1}(p, q ; n) C_{2}(p+q ; n)\right)^{-1}$.

Proof. Let $\omega$ and $\varphi$ be the harmonic forms representing $D(a)$ and $D(b)$ respectively. By applying in succession Schwarz's inequality, (1), and Lemma 1, we have

$$
\begin{aligned}
\|D(a)\|\|D(b)\| & =\left(\int_{M}|\omega|^{2} v_{g}\right)^{1 / 2}\left(\int_{M}|\varphi|^{2} v_{g}\right)^{1 / 2} \\
& \geq \int_{M}|\omega||\varphi| v_{g} \geq C_{1}^{-1}(p, q ; n) \int_{M}|\omega \wedge \varphi| v_{g} \\
& \geq C_{1}^{-1}(p, q ; n) C_{2}^{-1}(p+q ; n)\|a \cdot b\|
\end{aligned}
$$

because $\omega \wedge \varphi$ is a form dual to the class $a \cdot b$ and $C_{2}(n-p-q ; n)=$ $C_{2}(p+q ; n)$.

COROllaRY 3. For every $a \in H_{n-p}(M, R)$

$$
(\operatorname{Vol}(M, g))^{1 / 2}\|D(a)\| \geq C_{4}(p ; n)\|a\|
$$

where $C_{4}(p ; n)=C_{2}^{-1}(p ; n)$. If equality holds then the pointwise norm of the harmonic form representing $D(a)$ is constant.

Proof. Let $\omega$ be the harmonic form representing $D(a)$. Then by Lemma 1 and Schwarz's inequality,

$$
\begin{aligned}
\|a\| & \leq C_{2}(p ; n) \int_{M}|\omega| v_{g} \\
& \leq C_{2}(p ; n)\left(\int_{M}|\omega|^{2} v_{g}\right)^{1 / 2}\left(\int_{M} 1^{2} v_{g}\right)^{1 / 2} \\
& =C_{2}(p ; n)\|D(a)\|(\operatorname{Vol}(M, g))^{1 / 2} .
\end{aligned}
$$

If equality holds then $|\omega|$ is constant by Schwarz's inequality.

The collars of $(M, g)$. From now on suppose $M$ is torsion free. Thus the integral homology $H_{*}(M, Z)$ and the integral cohomology $H^{*}(M, Z)$ may be identified with subsets of $H_{*}(M, R)$ and $H^{*}(M, R)$ respectively which form lattices of these real vector spaces. 
The $p$-dimensional collar $\operatorname{Col}_{p}(M, g)$ of $(M, g)$ is defined by

$$
\mathrm{Col}_{p}(M, g)=\inf \left\{\|a\|: a \in H_{p}(M, Z), a \neq 0\right\} \text {. }
$$

REMARK. $\operatorname{Col}_{n}(M, g)=\operatorname{Vol}(M, g)$.

Let $\operatorname{det}\left(H^{p}(M, Z)\right)$ denote the determinant of the lattice $H^{p}(M, Z)$ in the inner product space $H^{p}(M, R)$. Recall the theorem of Minkowski which states

$$
\operatorname{det}\left(H^{p}(M, Z)\right) \geq u\left(b_{p}\right) \lambda^{b_{p}}
$$

where $\lambda=\min \left\{\|\alpha\|: \alpha \in H^{p}(M, Z), \alpha \neq 0\right\}$ and where $u\left(b_{p}\right)$ is a positive constant related to the Hermite constant depending only on the $p$ th Betti number $b_{p}$ of $M$. We will have need of the following inequality [4].

LEMMA 4. $\operatorname{det}\left(H^{p}(M, Z)\right) \geq u\left(b_{p}\right) \lambda_{1} \cdots \lambda_{b_{p}}$ where $\lambda_{1} \leq \cdots \leq \lambda_{b_{p}}$ are the successive minimums of the lattice $H^{p}(M, Z)$.

For notational convenience we set $h(k)=(u(k))^{1 / k}$.

We say $M$ satisfies the dual lattice condition in degree $p$ if for all $\alpha \in H^{p}(M, R),(\alpha \cup \beta)[M] \in Z$ for every $\beta \in H^{n-p}(M, Z)$ implies $\alpha \in$ $H^{p}(M, Z)$. This condition is important because of the following lemma.

LEMMA 5. (Berger [2].) If $M$ satisfies the dual lattice condition in degree $p$, then

$$
\operatorname{det}\left(H^{p}(M, Z)\right) \operatorname{det}\left(H^{n-p}(M, Z)\right)=1 .
$$

This condition is satisfied in all degrees by compact orientable surfaces, the product of spheres, the complex and quaternionic projective spaces, and the Cayley plane. Details may be found in [2]. Similar computations to those in [2] show that $C P^{m} \times C P^{n}, H P^{m} \times H P^{n}$, and $O P^{2} \times O P^{2}$ also satisfy the dual lattice condition in all degrees.

Proposition 6. Suppose $M$ satisfies the dual lattice condition in degree $p$, then

$$
\operatorname{Vol}(M, g) \geq C_{4}^{2}(p ; n) h^{2}\left(b_{p}\right) \operatorname{Col}_{p}(M, g) \operatorname{Col}_{n-p}(M, g) .
$$

Proof. Let $\alpha \in H^{p}(M, Z)$ and $\beta \in H^{n-p}(M, Z)$ be the minimum lattice points. Then $D(\alpha) \in H_{n-p}(M, Z)$ and $D(\beta) \in H_{p}(M, Z)$ are both non-zero. Hence by Corollary 3 and the definition of the collars of 
(M, g),

$$
\begin{aligned}
(\operatorname{Vol}(M, g))^{1 / 2}\|\alpha\| & \geq C_{4}(p ; n)\|D(\alpha)\| \\
& \geq C_{4}(p ; n) \operatorname{Col}_{n-p}(M, g)
\end{aligned}
$$

and

$$
\begin{gathered}
(\operatorname{Vol}(M, g))^{1 / 2}\|\beta\| \geq C_{4}(n-p ; n)\|D(\beta)\| \\
\geq C_{4}(n-p ; n) \operatorname{Col}_{p}(M, g) .
\end{gathered}
$$

By Lemmas 4 and 5,

$$
\begin{aligned}
1 & =\operatorname{det}\left(H^{p}(M, Z)\right) \operatorname{det}\left(H^{n-p}(M, Z)\right) \\
& \geq u\left(b_{p}\right)\|\alpha\|^{b_{p}} u\left(b_{n-p}\right)\|\beta\|^{b_{n-p}}
\end{aligned}
$$

from which follows the inequality

$$
1 \geq h^{2}\left(b_{p}\right)\|\alpha\|\|\beta\|
$$

by taking $b_{p}$ roots since $b_{p}=b_{n-p}$. Therefore

$$
\begin{aligned}
\operatorname{Vol}(M, g) & \geq \operatorname{Vol}(M, g) h^{2}\left(b_{p}\right)\|\alpha\|\|\beta\| \\
& \geq h^{2}\left(b_{p}\right) C_{4}(p ; n) C_{4}(n-p ; n) \operatorname{Col}_{p}(M, g) \operatorname{Col}_{n-p}(M, g) \\
& \geq h^{2}\left(b_{p}\right) C_{4}^{2}(p ; n) \operatorname{Col}_{p}(M, g) \operatorname{Col}_{n-p}(M, g)
\end{aligned}
$$

since $C_{4}(n-p ; n)=C_{4}(p ; n)$.

Proof of Theorem A. Let $(M, g)$ be a compact oriented $n$-dimensional Riemannian manifold with $H_{1}(M, Z) \cong Z$. Since $M$ is oriented, $H_{n-1}(M, Z)$ is torsion-free. Thus it is evident that $M$ satisfies the dual lattice condition in degree one. Thus by Proposition 6

$$
\operatorname{Vol}(M, g) \geq \operatorname{Col}_{1}(M, g) \operatorname{Col}_{n-1}(M, g)
$$

because $h(1)=C_{4}(1 ; n)=1$.

Suppose $f: M \rightarrow R / L Z$ is a Riemannian submersion onto the circle of length $L$ such that every level surface (or fiber) is a connected minimal hypersurface of $(M, g)$. Let $\omega=f^{*}(d t)$ where $d t$ is the canonical volume form on the circle $R / L Z$. Then $|\omega|=1$ because $f$ is a Riemannian submersion. Thus $* \omega=* \omega /|\omega|$ restricts to the volume form on each fiber of $f$. Hence a simple calculation shows that $d(* \omega)=\eta \wedge * \omega$ where $\eta$ is the 1 -form defined by $\eta(X)=-g(H, X), H$ being the mean curvature vector of the fibers of $f$. Consequently, $d(* \omega)=0$ because the fibers are 
minimal submanifolds. Hence all the fibers have the same volume $V$. Because $f$ is a Riemannian submersion, we may calculate $\operatorname{Vol}(M, g)$ by first integrating over the fibers and then over the circle $R / L Z$. Therefore $\operatorname{Vol}(M, g)=L V$.

Let $a \in H_{1}(M, Z) \cong Z$ be a generator. Then, because the fibers of $f$ are connected, $f_{*} a$ generates $H_{1}(R / L Z, Z)$. Thus if $s$ is a closed curve representing $a$,

$$
\left|\int_{s} \omega\right|=\left|\int_{f \circ s} d t\right|=L .
$$

If $T$ is any current in the homology class $a$, then $\int_{s}$ and $T$ are homologous, i.e.

$$
\int_{s}-T=\partial S
$$

for some 2-dimensional current $S$. Thus

$$
\int_{s} \omega-T(\omega)=\partial S(\omega)=S(d \omega)=S(0)=0
$$

Therefore, because $\|\omega\|_{0}=1\left(\right.$ since $\left.|\omega|_{0}=|\omega|=1\right)$

$$
|T| \geq|T(\omega)|=\left|\int_{s} \omega\right|=L \text {. }
$$

Consequently, $\operatorname{Col}_{1}(M, g) \geq L$.

Let $b \in H_{n-1}(M, Z) \cong Z$ be a generator and $T$ a current in the homology class $b$. Since the fibers of $f$ also represent $b$, integration over any fiber $W$ is homologous to $T$. Therefore, since $d(* \omega)=0$,

$$
T(* \omega)=\int_{W} * \omega=V
$$

But $|* \omega|_{0}=|* \omega|=|\omega|=1$. Hence $|T| \geq|T(* \omega)|=V$. Consequently, $\mathrm{Col}_{n-1}(M, g) \geq V$. Therefore

$$
\operatorname{Col}_{1}(M, g) \operatorname{Col}_{n-1}(M, g) \geq L V=\operatorname{Vol}(M, g) \text {. }
$$

Since we have already proved the reverse inequality we conclude $\mathrm{Col}_{1}(M, g)=L, \quad \mathrm{Col}_{n-1}(M, g)=V$, and $\operatorname{Vol}(M, g)=$ $\mathrm{Col}_{1}(M, g) \mathrm{Col}_{n-1}(M, g)$.

Conversely, suppose $\operatorname{Vol}(M, g)=\operatorname{Col}_{1}(M, g) \operatorname{Col}_{n-1}(M, g)$. Let $\omega$ be the harmonic 1-form representing a generator $\alpha \in H^{1}(M, Z)$. Then $|\omega|$ is constant by Corollary 3 because by the proof of Proposition 6, if equality 
holds in Proposition 6, then it holds in Corollary 3. Therefore $\varphi=\omega /|\omega|$ is a harmonic 1 -form with $|\varphi|=1$. Since $H_{1}(M, Z) \cong Z$, the periods of $\varphi$ are all integral multiples of a fixed number $L>0$. (In fact $L=|\omega|^{-1}$.) Thus we may define a map $f: M \rightarrow R / L Z$ such that $f^{*}(d t)=\varphi$ by first fixing a point $x_{0}$ in $M$ and then defining $f(x)$ to be the value modulo $L$ of the integral of $\varphi$ along any path joining $x_{0}$ to $x$. (See [2].) $f$ is a Riemannian submersion because $\left|f^{*}(d t)\right|=|\varphi|=1$. Consequently $* \varphi$ restricts to the volume form on the fibers of $f$. Therefore, because $d(* \varphi)=0$ since $\varphi$ is harmonic, the fibers of $f$ are all minimal submanifolds of $M$. Furthermore, they must be connected since each fiber represents the generator $D(\alpha)$ of $H_{n-1}(M, Z)$. Finally the first part of the proof shows that $L=\operatorname{Col}_{1}(M, g)$ and that $\operatorname{Col}_{n-1}(M, g)$ is the volume of a fiber.

Fully non-degenerate bilinear maps. Let $E, F$, and $W$ be three finite-dimensional real vector spaces, and let $B: E \times F \rightarrow W$ be a bilinear map. Choose bases $e_{1}, \ldots, e_{l}$ of $E$ and $f_{1}, \ldots, f_{k}$ of $F$. Thus $l=\operatorname{dim}(E)$ and $k=\operatorname{dim}(F)$. We will assume $l \leq k$. Form the $l \times k$ matrix $A=$ $\left(B\left(e_{l}, f_{j}\right)\right)$ whose entries are elements of $W$. The set of $l \times l$ submatrices of $A$ formed by deleting $k-l$ columns can be indexed by the set $\Pi$ consisting of all subsets $\pi$ of $\{1,2,3, \ldots, k\}$ of cardinality $l$ so that the submatrix $A_{\pi}$ is obtained by deleting the $j$ th column if and only if $j \notin \pi$. There are $\left(\begin{array}{l}k \\ l\end{array}\right)$ such matrices.

If we consider the entries of $A_{\pi}$ to be in the real symmetric algebra over $W$, we can form the $\operatorname{determinant} \operatorname{det}\left(A_{\pi}\right)$ which is a homogeneous element of degree $l$ in the symmetric algebra.

Suppose the set $\left\{\operatorname{det}\left(A_{\pi}\right): \pi \in \Pi\right\}$ is linearly independent over $R$ in the symmetric algebra. It is a simple matter to show that this condition is independent of the choice of bases of $E$ and $F$. For first suppose $e_{1}^{\prime}, \ldots, e_{l}^{\prime}$ is a second basis of $E$ related to $e_{1}, \ldots, e_{l}$ in one of the following three ways:

Case 1.

$$
e_{i}^{\prime}= \begin{cases}e_{i} & i \neq i_{0} \\ c e_{i_{0}} & i=i_{0}\end{cases}
$$

Case 2.

$$
e_{l}^{\prime}= \begin{cases}e_{i} & i \neq i_{0} \\ e_{t_{0}}+c e_{J_{0}} & i=i_{0}\end{cases}
$$


Case 3.

$$
e_{i}^{\prime}= \begin{cases}e_{i} & i \neq i_{0}, j_{0} \\ e_{j_{0}} & i=i_{0} \\ e_{i_{0}} & i=j_{0} .\end{cases}
$$

Thus, if we let $A^{\prime}$ be the matrix $\left(B\left(e_{i}^{\prime}, f_{j}\right)\right)$ and $A_{\pi}^{\prime}$ be the submatrix corresponding to $\pi \in \Pi$, then, by the properties of the determinant, we have:

Case 1. $\operatorname{det}\left(A_{\pi}^{\prime}\right)=c \operatorname{det}\left(A_{\pi}\right)$

Case 2. $\operatorname{det}\left(A_{\pi}\right)=\operatorname{det}\left(A_{\pi}\right)$

Case 3. $\operatorname{det}\left(A_{\pi}\right)=-\operatorname{det}\left(A_{\pi}\right)$.

Clearly $\left\{\operatorname{det}\left(A_{\pi}\right): \pi \in \Pi\right\}$ is linearly independent in every case.

Now suppose $f_{1}^{\prime}, \ldots, f_{k}^{\prime}$ is a second basis of $E$ related to $f_{1}, \ldots, f_{k}$ in one of the following three ways:

Case 1.

$$
f_{i}^{\prime}= \begin{cases}f_{i} & i \neq i_{0} \\ c f_{i_{0}} & i=i_{0}\end{cases}
$$

Case 2.

$$
f_{i}^{\prime}= \begin{cases}f_{i} & i \neq i_{0} \\ f_{i_{0}}+c f_{j_{0}} & i=i_{0}\end{cases}
$$

Case 3.

$$
f_{i}^{\prime}= \begin{cases}f_{i} & i \neq i_{0}, j_{0} \\ f_{j_{0}} & i=i_{0} \\ f_{i_{0}} & i=j_{0} .\end{cases}
$$

Thus, if we let $A^{\prime}$ be the matrix $\left(B\left(e_{i}, f_{j}^{\prime}\right)\right)$ and $A_{\pi}^{\prime}$ be the submatrix corresponding to $\pi \in \Pi$, then, by the properties of determinants, we have:

Case 1.

$$
\operatorname{det}\left(A_{\pi}^{\prime}\right)= \begin{cases}c \operatorname{det}\left(A_{\pi}\right) & \text { if } i_{0} \in \pi \\ \operatorname{det}\left(A_{\pi}\right) & \text { if } i_{0} \notin \pi\end{cases}
$$

Case 2.

$$
\operatorname{det}\left(A_{\pi}^{\prime}\right)= \begin{cases}\operatorname{det}\left(A_{\pi}\right) & \text { if either } i_{0} \notin \pi \text { or } \\ & \text { both } i_{0}, j_{0} \in \pi \\ \operatorname{det}\left(A_{\pi}\right)+c \operatorname{det}\left(A_{\pi^{\prime}}\right) & \text { if } i_{0} \in \pi, j_{0} \notin \pi \\ & \text { and } \pi^{\prime}=\pi \cup\left\{j_{0}\right\}-\left\{i_{0}\right\} .\end{cases}
$$


Case 3.

$$
\operatorname{det}\left(A_{\pi}^{\prime}\right)= \begin{cases}\operatorname{det}\left(A_{\pi}\right) & \text { if } i_{0}, j_{0} \notin \pi \\ -\operatorname{det}\left(A_{\pi}\right) & \text { if } i_{0}, j_{0} \in \pi \\ \operatorname{det}\left(A_{\pi^{\prime}}\right) & \text { if } i_{0} \in \pi, j_{0} \notin \pi \\ & \text { and } \pi^{\prime}=\pi \cup\left\{j_{0}\right\}-\left\{i_{0}\right\} \\ \operatorname{det}\left(A_{\pi^{\prime}}\right) & \text { if } i_{0} \notin \pi, j_{0} \in \pi \\ & \text { and } \pi^{\prime}=\pi \cup\left\{i_{0}\right\}-\left\{j_{0}\right\}\end{cases}
$$

Once again, in every case, $\left\{\operatorname{det}\left(A_{\pi}^{\prime}\right): \pi \in \Pi\right\}$ is linearly independent.

Since any two pairs of bases for $E$ and $F$ are related to each other by a sequence of the elementary changes of bases just described, we see that $\left\{\operatorname{det}\left(A_{\pi}\right): \pi \in \Pi\right\}$ is linearly independent for every pair of bases for $E$ and $F$ if it is for one such pair. We will say $B$ is fully non-degenerate if the set $\left\{\operatorname{det}\left(A_{\pi}\right): \pi \in \Pi\right\}$ is linearly independent in the symmetric algebra over $W$.

Suppose $B$ is fully non-degenerate. Then for any bases $e_{1}, \ldots, e_{l}$ of $E$ and $f_{1}, \ldots, f_{k}$ of $F$ and for every $\pi \in \Pi$, $\operatorname{det}\left(A_{\pi}\right) \neq 0$. Recall that $\operatorname{det}\left(A_{\pi}\right)$ is the sum of terms where each term is \pm 1 times a product of $l$ entries chosen from $A_{\pi}$ in such a way that an entry has been chosen from each row and column exactly once. Since $\operatorname{det}\left(A_{\pi}\right) \neq 0$, one of these terms must be non-zero. Thus we are able to choose $l$ non-zero entries from $A_{\pi}$ such that any entry has been chosen from each row and column exactly once. Therefore, doing this for each $\pi$, we can choose $\left(\begin{array}{l}k \\ l\end{array}\right) l$ non-zero entries from $A$ so that an entry has been chosen exactly $\left(\begin{array}{l}k \\ l\end{array}\right)$-times from each row and exactly $\left(\begin{array}{c}k-1 \\ l-1\end{array}\right)$-times from each column. Some entries may have been chosen more than once.

Lemma 7. Suppose $\cup . H^{p}(M, R) \times H^{q}(M, R) \rightarrow H^{p+q}(M, R)$ with $p+q \leq n$ is fully non-degenerate. Then

$$
\begin{aligned}
\left(\operatorname{det}\left(H^{p}(M, Z)\right)\right)^{\left(1 / b_{p}\right)}(\operatorname{det} & \left.\left(H^{q}(M, Z)\right)\right)^{\left(1 / b_{p}\right)} \\
& \geq h\left(b_{p}\right) h\left(b_{q}\right) C_{3}(p, q ; n) \operatorname{Col}_{n-p-q}(M, g) .
\end{aligned}
$$

Proof. Let $b_{p}=l, b_{q}=k$, and assume $l \leq k$. Let $\alpha_{1}, \ldots, \alpha_{l}$ and $\beta_{1}, \ldots, \beta_{k}$ be bases of $H^{p}(M, R)$ and $H^{q}(M, R)$ respectively which are the successive minimums of the lattices $H^{p}(M, Z)$ and $H^{q}(M, Z)$. Since $\checkmark$ is fully non-degenerate, we can choose $\left({ }_{l}^{k}\right) l$ pairs $\left(\alpha_{i}, \beta_{j}\right)$ with $\alpha_{i} \cup \beta_{j} \neq 0$ so that each $\alpha_{i}$ is a member of a pair exactly $\left(\begin{array}{l}k \\ l\end{array}\right)$-times and 
each $\beta_{j}$ is a member of a pair exactly $\left(\begin{array}{c}k-1 \\ l-1\end{array}\right)$-times. For each pair $\left(\alpha_{i}, \beta_{J}\right)$, $0 \neq D\left(\alpha_{i}\right) \cdot D\left(\beta_{j}\right) \in H_{n-p-q}(M, Z)$. Thus, by Lemma 2,

$$
\left\|\alpha_{i}\right\|\left\|\beta_{j}\right\| \geq C_{3}(p, q ; n) \mathrm{Col}_{n-p-q}(M, g) .
$$

Multiplying all $\left(\begin{array}{l}k \\ l\end{array}\right) l$ of these inequalities together gives

$$
\begin{aligned}
& \left(\left\|\alpha_{1}\right\| \cdots\left\|\alpha_{l}\right\|\right)^{\left(\begin{array}{c}
k \\
l
\end{array}\right)}\left(\left\|\beta_{1}\right\| \cdots\left\|\beta_{k}\right\|\right)^{\left(\begin{array}{c}
k-1 \\
l-1
\end{array}\right)} \\
& \quad \geq\left(C_{3}(p, q ; n) \mathrm{Col}_{n-p-q}(M, g)\right)^{\left(\begin{array}{c}
k \\
l
\end{array}\right) l} .
\end{aligned}
$$

Thus, by Lemma 4,

$$
\begin{aligned}
& \left(\operatorname{det}\left(H^{p}(M, Z)\right)\right)^{\left({ }^{k}\right)}\left(\operatorname{det}\left(H^{q}(M, Z)\right)\right)^{\left(\begin{array}{c}
k-1 \\
l-1
\end{array}\right)} \\
& \quad \geq u(l)^{\left({ }^{k}\right)} u(k)^{\left({ }_{l}^{k-1}\right)}\left(C_{3}(p, q ; n) \operatorname{Col}_{n-p-q}(M, g)\right)^{\left({ }_{l}^{k}\right) l} .
\end{aligned}
$$

The desired inequality follows by taking $\left(\begin{array}{l}k \\ l\end{array}\right) l$-roots.

Proposition 8. Suppose $M$ satisfies the dual lattice condition in degree $p$ for all $0<p<n$, and suppose $\cup: H^{p}(M, R) \times H^{q}(M, R) \rightarrow$ $H^{p+q}(M, R)$ is fully non-degenerate for all $p, q$ with $p+q \leq n$. Then, given any positive integers $i_{1}, \ldots, i_{k}$ such that $i_{1}+\cdots+i_{k}=n$, there is a constant $C_{M}\left(i_{1}, \ldots, i_{k}\right)>0$ such that

$$
\operatorname{Vol}(M, g) \geq C_{M}\left(i_{1}, \ldots, i_{k}\right) \operatorname{Col}_{\iota_{1}}(M, g) \cdots \operatorname{Col}_{i_{k}}(M, g)
$$

for every Riemannian metric $g$ on $M$.

Proof. Set $C(p, q)=h\left(b_{p}\right) h\left(b_{q}\right) C_{3}(p, q ; n)$ and $H^{p}=H^{p}(M, R)$. Then by applying Lemma $7 k$ times,

$$
\begin{aligned}
& \left(\operatorname{det}\left(H^{0}\right)\right)^{b_{0}^{-1}}\left(\operatorname{det}\left(H^{n-i_{1}}\right)\right)^{b_{n-l_{1}}^{-1}} \geq C\left(0, n-i_{1}\right) \operatorname{Col}_{l_{1}}(M, g) \\
& \left(\operatorname{det}\left(H^{i_{1}}\right)\right)^{b_{t_{1}}^{-1}}\left(\operatorname{det}\left(H^{n-i_{1}-i_{2}}\right)\right)^{b_{n-t_{1}-t_{2}}^{-1}} \geq C\left(i_{1}, n-i_{1}-i_{2}\right) \operatorname{Col}_{t_{2}}(M, g) \\
& \left(\operatorname{det}\left(H^{n-i_{k}}\right)\right)^{b_{n-i_{k}}^{-1}}\left(\operatorname{det}\left(H^{0}\right)\right)^{b_{0}^{-1}} \leq C\left(n-i_{k}, 0\right) \operatorname{Col}_{i_{k}}(M, g) .
\end{aligned}
$$

Since $\operatorname{det}\left(H^{0}\right)=(\operatorname{Vol}(M, g))^{1 / 2}$ and $b_{p}=b_{n-p}$, the proof is completed after multiplying these inequalities and applying Lemma 5.

REMARK. This is a version of the stable isosystolic inequality. It bears comparison with the stable isosystolic inequality 7.4.C in Gromov [7]. In fact his result implies ours. For if $M$ satisfies the hypothesis of Proposition 8 and $i_{1}, \ldots, i_{k}$ are positive integers satisfying $i_{1}+\cdots+i_{k}=n$, then 
the hypothesis that the cup products are fully non-degenerate implies that the cup product form $H^{i_{1}}(M) \times \cdots \times H^{i_{k}}(M) \rightarrow H^{n}(M)$ is non-degenerate in the sense of $\$ 7.4$ of [7]. Thus Gromov's result 7.4.C gives a constant $C$ and non-zero homology classes $h_{j}$ in $H_{i_{j}}(M, Z)$ such that $\operatorname{Vol}_{R}\left(h_{1}\right) \cdots \operatorname{Vol}_{R}\left(h_{k}\right) \leq C \operatorname{Vol}(M, g)$. Since $\operatorname{Col}_{i_{j}}(M, g) \leq \operatorname{Vol}_{R}\left(h_{j}\right)$ Proposition 8 follows. However we will prove Theorem B by showing the cohomology ring of $C P^{m} \times C P^{n}$ satisfies the hypothesis of Proposition 8 .

Proof of Theorem B. Let $M=C P^{m} \times C P^{n}$ with $m \geq n . H^{*}(M, R)$ is generated as an algebra by two elements $x, y \in H^{2}(M, R)$ satisfying $x y=y x, x^{m+1}=0$ and $y^{n+1}=0$. Thus $H^{2 p}(M, R)$ has a basis consisting of

$$
\begin{array}{ll}
x^{p}, x^{p-1} y, x^{p-2} y^{2}, \ldots, y^{p} & \text { if } p<n, \\
x^{p}, x^{p-1} y, x^{p-2} y^{2}, \ldots, x^{p-n} y^{n} & \text { if } n \leq p \leq m, \text { or } \\
x^{m} y^{p-m}, x^{m-1} y^{p-m-1}, \ldots, x^{p-n} y^{n} & \text { if } p>m .
\end{array}
$$

Thus if $e_{1}, \ldots, e_{l}$ is this basis for $H^{2 p}(M, R)$ and $f_{1}, \ldots, f_{k}$ is this basis for $H^{2 q}(M, R)$ where we assume $l=b_{2 p} \leq k=b_{2 q}$ and $p+q \leq m+n$, then $e_{i} \cup f_{j}=e_{i^{\prime}} \cup f_{j^{\prime}}$ whenever $i+j=i^{\prime}+j^{\prime}$. Hence, if we let $z_{i+j}=e_{i} \cup f_{j}$, then the matrix $A$ of the bilinear map $\cup: H^{2 p}(M, R) \times$ $H^{2 q}(M, R) \rightarrow H^{2 p+2 q}(M, R)$ is of the form

$$
\left(\begin{array}{ccccc}
z_{2} & z_{3} & z_{4} & \cdots & z_{k+1} \\
z_{3} & z_{4} & z_{5} & \cdots & z_{k+2} \\
z_{4} & z_{5} & z_{6} & \cdots & z_{k+3} \\
\vdots & \vdots & \vdots & & \vdots \\
z_{l+1} & z_{l+2} & z_{l+3} & \cdots & z_{l+k}
\end{array}\right) .
$$

The $z_{i}$ are distinct elements of the basis for $H^{2 p+2 q}(M, R)$ except that the first few $z_{i}$ 's and the last few, namely $z_{2}, \ldots, z_{l}$ and $z_{k+2}, \ldots, z_{l+k}$, may possibly be zero when $p+q$ is large enough. In any case, $z_{l+1}, z_{l+2}, \ldots, z_{l+k}$ are linearly independent elements of $H^{2 p+2 q}(M, R)$.

Again recall that the determinant of a square matrix is the sum of terms where each term is \pm 1 times a product of entries chosen from the matrix so that an entry is chosen once from each row and each column. For $\pi \in \Pi$, we break down the determinant of $A_{\pi}$ as follows:

$$
\operatorname{det}\left(A_{\pi}\right)=D_{\pi}+E_{\pi}
$$

where $D_{\pi}$ is the sum of the terms involving only $z_{l+1}, \ldots, z_{k+1}$, and $E_{\pi}$ is the sum of the terms that contain at least one of $z_{2}, \ldots, z_{l}, z_{k+2}, \ldots, z_{l+k}$. We will prove the $\operatorname{set}\left\{\operatorname{det}\left(A_{\pi}\right): \pi \in \Pi\right\}$ to be linearly independent by 
first proving that $\left\{\operatorname{det}\left(A_{\pi}\right): \pi \in \Pi\right\}$ is linearly independent if $\left\{D_{\pi}\right.$ : $\pi \in \Pi\}$ is linearly independent, and then by proving that $\left\{D_{\pi}: \pi \in \Pi\right\}$ is linearly independent.

Suppose $\left\{D_{\pi}: \pi \in \Pi\right\}$ is linearly independent, and let

$$
\sum a_{\pi} \operatorname{det}\left(A_{\pi}\right)=0 \text {. }
$$

Thus

$$
\sum a_{\pi} D_{\pi}+\sum a_{\pi} E_{\pi}=0 .
$$

But $\sum a_{\pi} D_{\pi}$ is contained in the subspace of the symmetric algebra over $H^{2 p+2 q}(M, R)$ spanned by simple homogeneous elements of degree $l$ involving only $z_{l+1}, \ldots, z_{k+1}$ while $\sum a_{\pi} E_{\pi}$ is contained in the subspace spanned by simple homogeneous elements of degree $l$ each of which contains at least one of $z_{2}, \ldots, z_{l}, z_{k+2}, \ldots, z_{l+k}$. Since these two subspaces have only zero in common.

$$
\sum a_{\pi} D_{\pi}=-\sum a_{\pi} E_{\pi}=0 .
$$

Hence, $a_{\pi}=0$ for all $\pi \in \Pi$ by the linear independence of the $D_{\pi}$. Therefore $\left\{\operatorname{det}\left(A_{\pi}\right): \pi \in \Pi\right\}$ is linearly independent.

We next prove $\left\{D_{\pi}: \pi \in \Pi\right\}$ is linearly independent by double induction on $l$ and $k$.

Clearly, $\left\{D_{\pi}: \pi \in \Pi\right\}$ is linearly independent if $l=1$ and $k \geq 1$ since $z_{2}, \ldots, z_{k+1}$ is linearly independent. Also, if $l=k,\left\{D_{\pi}: \pi \in \Pi\right\}=$ $\left\{\left(z_{l+1}\right)^{l}\right\}$ is linearly independent because $z_{l+1} \neq 0$.

Now suppose that $1<l<k$ and that we have linear independence for matrices of dimensions $(l-1) \times(k-1)$ and $l \times(k-1)$. Decompose $\Pi$ into two disjoint subsets

$$
\Pi=\Pi_{0} \cup \Pi_{1}
$$

where $\Pi_{0}$ is the set of all subsets of $\{1,2, \ldots, k-1\}$ of cardinality $l$, and $\Pi_{1}$ is the set of all subsets of $\{1,2, \ldots, k\}$ of cardinality $l$ which contain $k$. $\Pi_{1}$ is in one-to-one correspondence with the set $\Pi^{\prime}$ of all subsets of $\{1,2, \ldots, k-1\}$ of cardinality $l-1$ where $\pi \in \Pi_{1}$ corresponds to $\pi^{\prime} \in$ $\Pi^{\prime}$ if and only if $\pi=\pi^{\prime} \cup\{k\}$.

Let $A^{0}$ be the $l \times(k-1)$ matrix

$$
\left(\begin{array}{cccc}
z_{2} & z_{3} & \ldots & z_{k} \\
z_{3} & z_{4} & \cdots & z_{k+1} \\
\vdots & \vdots & & \vdots \\
z_{l+1} & z_{l+2} & \cdots & z_{k+l-1}
\end{array}\right)
$$


obtained from $A$ by deleting the last column. Then, for $\pi \in \Pi_{0}$ let

$$
\operatorname{det}\left(A_{\pi}^{0}\right)=D_{\pi}^{0}+E_{\pi}^{0}
$$

where $D_{\pi}^{0}$ is the sum of the terms involving only $z_{l+1}, \ldots, z_{k}$. By induction $\left\{D_{\pi}^{0}: \pi \in \Pi_{0}\right\}$ is linearly independent. Observe also that if $\pi \in \Pi_{0}$, then

$$
D_{\pi}=D_{\pi}^{0}+F_{\pi}
$$

where $D_{\pi}^{0}$ is as above and $F_{\pi}$ is the sum of the terms in $D_{\pi}$ which contain $z_{k+1}$.

Let $A^{1}$ be the $(l-1) \times(k-1)$ matrix

$$
\left(\begin{array}{cccc}
z_{3} & z_{4} & \cdots & z_{k} \\
z_{4} & z_{5} & \cdots & z_{k+1} \\
\vdots & \vdots & & \vdots \\
z_{l+1} & z_{l+2} & \cdots & z_{k+l-1}
\end{array}\right)
$$

obtained from $A$ by deleting the last column and the first row. For $\pi^{\prime} \in \Pi^{\prime}$, let

$$
\operatorname{det}\left(A_{\pi^{\prime}}^{1}\right)=D_{\pi^{\prime}}^{\prime}+E_{\pi^{\prime}}^{\prime}
$$

where $D_{\pi^{\prime}}^{\prime}$ is the sum of the terms involving only $z_{l+1}, \ldots, z_{k}$ and $E_{\pi^{\prime}}^{\prime}$ are the other terms. By induction, $\left\{D_{\pi^{\prime}}^{\prime}: \pi^{\prime} \in \Pi^{\prime}\right\}$ is linearly independent. Observe that if $\pi \in \Pi_{1}$ corresponds to $\pi^{\prime} \in \Pi^{\prime}$, then

$$
D_{\pi}=z_{k+1}\left(D_{\pi^{\prime}}^{\prime}+F_{\pi^{\prime}}^{\prime}\right)
$$

where the terms in $F_{\pi^{\prime}}^{\prime}$ must contain $z_{k+1}$. If we set $D_{\pi}^{1}=\left(D_{\pi^{\prime}}^{\prime}+F_{\pi^{\prime}}^{\prime}\right)$, then $D_{\pi}=z_{k+1} D_{\pi}^{1}$ and the set $\left\{D_{\pi}^{1}: \pi \in \Pi_{1}\right\}$ is linearly independent. The linear independence of the $D_{\pi}^{1}$ follows easily from the linear independence of the $D_{\pi^{\prime}}^{\prime}$ and the fact that the $D_{\pi^{\prime}}^{\prime}$ lie in the subspace of the symmetric algebra over $H^{2 p+2 q}(M, R)$ spanned by simple homogeneous elements of degree $l-1$ involving only $z_{l+1}, \ldots, z_{k}$ while the $F_{\pi^{\prime}}^{\prime}$ lie in the subspace spanned by the simple homogeneous elements that involve $z_{k+1}$. In short, the $D_{\pi^{\prime}}^{\prime}$ and the $F_{\pi^{\prime}}^{\prime}$ lie in two different subspaces which have only zero in common.

Now, suppose

$$
\sum_{\pi \in \Pi_{0}} a_{p} D_{\pi}+\sum_{\pi \in \Pi_{1}} a_{\pi} D_{\pi}=0
$$

Then

$$
\sum_{\pi \in \Pi_{0}} a_{\pi} D_{\pi}^{0}+\sum_{\pi \in \Pi_{0}} a_{\pi} F_{p}+\sum_{\pi \in \Pi_{1}} a_{\pi} z_{k+1} D_{\pi}^{1}=0
$$


But the first term of the last equation lies in the subspace spanned by terms involving only $z_{l+1}, \ldots, z_{k}$ whereas the last two terms lie in the subspace spanned by terms which all involve $z_{k+1}$. Since these two subspaces have only zero in common,

$$
\sum_{\pi \in \Pi_{0}} a_{\pi} D_{\pi}^{0}=0=\sum_{\pi \in \Pi_{0}} a_{\pi} F_{\pi}+\sum_{\pi \in \Pi_{1}} a_{\pi} z_{k+1} D_{\pi}^{1} .
$$

Since the $D_{\pi}^{0}$ are linearly independent, $a_{\pi}=0$ for all $\pi \in \Pi_{0}$. Therefore

$$
\sum_{\pi \in \Pi_{1}} a_{\pi} z_{k+1} D_{\pi}^{1}=0
$$

Therefore

$$
\sum_{\pi \in \Pi_{1}} a_{\pi} D_{\pi}^{1}=0
$$

Therefore $a_{\pi}=0$ for all $\pi \in \Pi_{1}$ since the $D_{\pi}^{1}$ are linearly independent. This completes the induction step proving that $\left\{D_{\pi}: \pi \in \Pi\right\}$ is linearly independent.

Therefore $\checkmark$ is fully non-degenerate. Hence Theorem B follows from Proposition 8.

Example. Proposition 8 does not apply to the product of more than two projective spaces. For example, let $M=C P^{2} \times C P^{2} \times C P^{2}$, and let $x, y, z \in H^{2}(M, R)$ be generators of $H^{*}(M, R)$ which satisfy $x^{3}=y^{3}=$ $z^{3}=0$ and commute. With respect to the basis $\left\{x^{2}, x y, y^{2}, x z, z^{2}, y z\right\}$ for $H^{4}(M, R)$ and the basis $\left\{x^{2} y, x^{2} z, x y^{2}, y^{2} z, x z^{2}, y z^{2}, x y z\right\}$ for $H^{6}(M, R)$ the bilinear map $\cup: H^{4}(M, R) \times H^{6}(M, R) \rightarrow H^{10}(M, R)$ has the matrix

$$
\left(\begin{array}{ccccccc}
0 & 0 & 0 & x^{2} y^{2} z & 0 & x^{2} y z^{2} & 0 \\
0 & 0 & 0 & 0 & x^{2} y z^{2} & x y^{2} z^{2} & x^{2} y^{2} z \\
0 & x^{2} y^{2} z & 0 & 0 & x y^{2} z^{2} & 0 & 0 \\
0 & 0 & x^{2} y^{2} z & x y^{2} z^{2} & 0 & 0 & x^{2} y z^{2} \\
x^{2} y z^{2} & 0 & x y^{2} z^{2} & 0 & 0 & 0 & 0 \\
x^{2} y^{2} z & x^{2} y z^{2} & 0 & 0 & 0 & 0 & x y^{2} z^{2}
\end{array}\right) .
$$

By direct calculation the submatrix formed by deleting the last column has determinant equal to zero. This shows that $\checkmark$ is not fully non-degenerate.

On the other hand, it seems safe to conjecture that the product of more than two complex projective spaces satisfies stable isosystolic inequalities. To prove this one would need to show that the cohomology 
ring of the product of several complex projective spaces satisfies the appropriate non-degeneracy conditions in the hypothesis of Gromov's version of the stable isosystolic inequality.

\section{The First Eigenvalue.}

Proposition 9. Let $\lambda_{1}(M, g)$ denote the first eigenvalue of the Laplacian of $(M, g)$. Suppose $M$ satisfies the dual lattice condition in degree 1 , then

$$
\lambda_{1}(M, g) \leq \frac{4 \pi^{2}(\operatorname{Vol}(M, g))^{2}}{\left(h\left(b_{1}\right)\right)^{8} \operatorname{Col}_{1}^{4}(M, g) \operatorname{Col}_{n-1}^{2}(M, g)}
$$

Proof. Set $\mu_{p}=\inf \left\{\|\alpha\|: \theta \in H^{p}(M, Z), \alpha \neq 0\right\}$. Berger [3] proves $\lambda_{1} \leq 4 \pi^{2} c^{-2} \mu_{1}^{4}$ where $c$ is the $(n-1)$-dimensional carcan of $(M, g)$ which he defines as the infimum of the volumes of all compact oriented ( $n-1)$-dimensional submanifolds of $M$ whose fundamental class is not null-homologous in $M$. Since integration over every such submanifold defines an $(n-1)$-dimensional current whose mass equals the volume of the submanifold, $\operatorname{Col}_{n-1}(M, g) \leq c$. Thus, by Lemma 4,

$$
\lambda_{1}(M, g) \operatorname{Col}_{n-1}^{2}(M, g) \leq 4 \pi^{2} \mu_{1}^{4} \leq 4 \pi^{2} \frac{\left(\operatorname{det}\left(H^{1}(M, Z)\right)\right)^{4 b_{1}^{-1}}}{\left(h\left(b_{1}\right)\right)^{4}} .
$$

Now by Corollary 3 and Lemma 4,

$$
\begin{aligned}
\operatorname{Col}_{1}(M, g) & \leq \mu_{n-1}(\operatorname{Vol}(M, g))^{1 / 2} \\
& \leq \frac{\left(\operatorname{det}\left(H^{n-1}(M, Z)\right)\right)^{b_{n-1}^{-1}}}{h\left(b_{n-1}\right)}(\operatorname{Vol}(M, g))^{1 / 2} .
\end{aligned}
$$

Raising the last inequality to the fourth power and multiplying it with the previous inequality gives

$$
\begin{aligned}
& \lambda_{1}(M, g) \operatorname{Col}_{1}^{4}(M, g) \operatorname{Col}_{n-1}^{2}(M, g) \\
& \leq 4 \pi^{2} \frac{\left(\operatorname{det}\left(H^{1}(M, Z)\right)\right)^{4 b_{1}^{-1}}}{\left(h\left(b_{1}\right)\right)^{4}} \cdot \frac{\left(\operatorname{det}\left(H^{n-1}(M, Z)\right)\right)^{4 b_{n-1}^{-1}}}{\left(h\left(b_{n-1}\right)\right)^{4}}(\operatorname{Vol}(M, g))^{2}
\end{aligned}
$$

which by Lemma 5 is equivalent to the inequality to be proved since $b_{1}=b_{n-1}$. 


\section{REFERENCES}

[1] Marcel Berger, Du Côté de chez Pu, Ann. Scient. Éc. Norm. Sup., 5 (1972), 1-44.

[2] _ A l'Ombre de Loewner, Ann. Scient. Éc. Norm. Sup., 5 (1972), 241-260.

[3] Sur les premières valeurs propres des variétés Riemanniennes, Compositio Math., 26 (1973), 129-149.

[4] J. W. S. Cassels, An Introduction to the Geometry of Numbers, Springer-Verlag, 1959.

[5] Herbert Federer, Geometric Measure Theory, Springer-Verlag, 1969.

[6] Mikhael Gromov, Structures métriques pour les variétés riemanniennes, Cedic/Fernand Nathan 1981.

[7] _ Filling Riemannian manifolds, J. Differential Geometry, 18 (1983), 1-148.

[8] P. M. Pu, Some inequalities in certain non-orientable manifolds, Pacific J. Math., 11 (1952), 55-71.

[9] Georges de Rham, Variétés Differentiables, Hermann, 1955.

[10] Frank W. Warner, Foundations of Differential Manifolds and Lie Groups, Scott, Foresman and Company, 1971.

[11] Hassler Whitney, Geometric Integration Theory, Princeton University Press, 1957.

Received June 27, 1984 and in revised form October 1, 1984.

ST. LOUIS UNIVERSITY

ST. LouIS, MO 63103 


\title{
PACIFIC JOURNAL OF MATHEMATICS EDITORS
}

V. S. VARADARAJAN (Managing Editor)
University of California
Los Angeles, CA 90024
HEBERT ClEMENS
University of Utah
Salt Lake City, UT 84112
CHARLES R. DEPRIMA
California Institute of Technology
Pasadena, CA 91125

R. FINN

Stanford University

Stanford, CA 94305

HERMANN FLASCHKA

University of Arizona

Tucson, AZ 85721

Ramesh A. Gangolli

University of Washington

Seattle, WA 98195

ROBION KIRBY

University of California

Berkeley, CA 94720
C. C. MOORE

University of California

Berkeley, CA 94720

H. SAMELSON

Stanford University

Stanford, CA 94305

HaRold Stark

University of California, San Diego

La Jolla, CA 92093

\section{ASSOCIATE EDITORS}

\author{
R. ARENS \\ E. F. BECKENBACH \\ B. H. NeumanN \\ F. Wolf \\ K. YoSHIDA \\ (1906-1982)
}

\section{SUPPORTING INSTITUTIONS}

UNIVERSITY OF ARIZONA
UNIVERSITY OF BRITISH COLUMBIA
CALIFORNIA INSTITUTE OF TECHNOLOGY
UNIVERSITY OF CALIFORNIA
MONTANA STATE UNIVERSITY
UNIVERSITY OF NEVADA, RENO
NEW MEXICO STATE UNIVERSITY
OREGON STATE UNIVERSITY

UNIVERSITY OF OREGON

UNIVERSITY OF SOUTHERN CALIFORNIA

STANFORD UNIVERSITY

UNIVERSITY OF HAWAII

UNIVERSITY OF TOKYO

UNIVERSITY OF UTAH

WASHINGTON STATE UNIVERSITY UNIVERSITY OF WASHINGTON

The Supporting Institutions listed above contribute to the cost of publication of this Journal, but they are not owners or publishers and have no responsibility for its content or policies.

Mathematical papers intended for publication in the Pacific Journal of Mathematics should be in typed form or offset-reproduced (not dittoed), double spaced with large margins. Please do not use built up fractions in the text of the manuscript. However, you may use them in the displayed equations. Underline Greek letters in red, German in green, and script in blue. The first paragraph must be capable of being used separately as a synopsis of the entire paper. In particular it should contain no bibliographic references. Please propose a heading for the odd numbered pages of less than 35 characters. Manuscripts, in triplicate, may be sent to any one of the editors. Please classify according to the scheme of Math. Reviews, Index to Vol. 39. Supply name and address of author to whom proofs should be sent. All other communications should be addressed to the managing editor, or Elaine Barth, University of California, Los Angeles, California 90024.

There are page-charges associated with articles appearing in the Pacific Journal of Mathematics. These charges are expected to be paid by the author's University, Government Agency or Company. If the author or authors do not have access to such Institutional support these charges are waived. Single authors will receive 50 free reprints; joint authors will receive a total of 100 free reprints. Additional copies may be obtained at cost in multiples of 50 .

The Pacific Journal of Mathematics is issued monthly as of January 1966. Regular subscription rate: $\$ 190.00$ a year (5 Vols., 10 issues). Special rate: $\$ 95.00$ a year to individual members of supporting institutions.

Subscriptions, orders for numbers issued in the last three calendar years, and changes of address should be sent to Pacific Journal of Mathematics, P.O. Box 969, Carmel Valley, CA 93924, U.S.A. Old back numbers obtainable from Kraus Periodicals Co., Route 100, Millwood, NY 10546.

The Pacific Journal of Mathematics at P.O. Box 969, Carmel Valley, CA 93924 (ISSN 0030-8730) publishes 5 volumes per year. Application to mail at Second-class postage rates is pending at Carmel Valley, California, and additional mailing offices. Postmaster: Send address changes to Pacific Journal of Mathematics, P.O. Box 969, Carmel Valley, CA 93924.

\section{PUBLISHED BY PACIFIC JOURNAL OF MATHEMATICS, A NON-PROFIT CORPORATION}

Copyright $\subset 1986$ by Pacific Journal of Mathematics 


\section{Pacific Journal of Mathematics}

\section{Vol. 121, No. 2 December, 1986}

Jorge Almeida, Minimal nonpermutative pseudovarieties of semigroups.

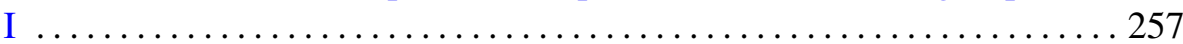

Jorge Almeida, Minimal nonpermutative pseudovarieties of semigroups.

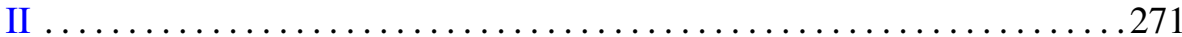

Carlos Andradas Heranz and José Manuel Gamboa Mutuberría, On

projections of real algebraic varieties $\ldots \ldots \ldots \ldots \ldots \ldots \ldots \ldots \ldots 281$

Zeev Ditzian, Inverse theorems for multidimensional Bernstein operators . . 293

M. Furi and Maria Patrizia Pera, A continuation principle for forced oscillations on differentiable manifolds $\ldots \ldots \ldots \ldots \ldots \ldots \ldots \ldots \ldots . \ldots . \ldots . \ldots 321$

James J. Hebda, The collars of a Riemannian manifold and stable

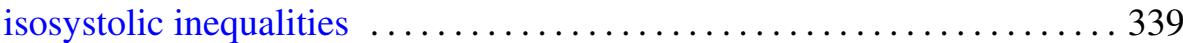

Henryk Hecht and Dragan Miličić, Character identities and asymptotic behavior of matrix coefficients of discrete series ................ 357

Piotr Jakóbczak, The boundary regularity of the solution of the $\bar{\partial}$-equation in the product of strictly pseudoconvex domains $\ldots \ldots \ldots \ldots \ldots \ldots . \ldots 371$

Krzysztof Jarosz, Isometries between injective tensor products of Banach

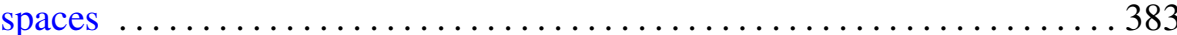

Hans Keller, On valued, complete fields and their automorphisms ........ 397

David Masser and Peter Man-Kit Shiu, On sparsely totient numbers . . . . 407

Tze-Beng Ng, Vector bundles over $(8 k+3)$-dimensional manifolds

Thomas Joseph Ransford, The spectrum of an interpolated operator and analytic multivalued functions

Akihito Uchiyama, On the radial maximal function of distributions 467 Jang-Mei Gloria Wu, On singularity of harmonic measure in space 